\title{
La ejecución del Programa Ampliado Materno-Infantil en el Estado Zulia y la actitud de sus beneficiarios
}

\section{Alvarado Chacín, Neritza*}

\section{Resumen}

Se analizan dos aspectos no previstos en la mayoria de los programas de la política social compensatoria tomando como caso de estudio la dinámica del Programa Ampliado Materno Infantil (PAMI) en el Estado Zulia (Venezuela). Tales aspectos son: 1) la evaluación del proceso de ejecución y 2) la actitud de sus beneficiarios. El primero se estudia según las siguientes variables: estructura organizativa; estrategia operativa; sistemas de información y difusión; mecanismos de supervisión, control y seguimiento; cobertura geográfica y social; recursos humanos, físicos y financieros; eficacia o cumplimiento de metas. La actitud se estudia a partir del nivel de conocimiento o de información que los beneficiarios tienen del programa; la opinión sobre el impacto del mismo en sus condiciones de vida; su reacción más frecuente ante cambios parciales o definitivos del programa, y las formas de participación en éste. El objetivo básico es insistir en la necesidad de diseñar y evaluar integralmente los programas de atención a la pobreza, incorporando estos aspectos, toda vez que inciden, directa o indirectamente, en el bajo impacto social de dicha política.

Palabras clave: Programas sociales compensatorios, proceso de ejecución, actitud de los beneficiarios, PAMI, Zulia, Venezuela.

Recibido: 98-02-06. Aceptado: 99-09-15

* Socióloga, Magister en Sociologia del Desarrollo, Protesora Asociada e Investigadora del Instituto de Investigaciones de la Facultad de Ciencias Económicas y Sociales de la Universidad del Zulia, Cursante del Doctorado en Estudios del Desarrollo del CENDES-UCV. Fax: 02751.16.63. E-m: nalvaradreacciun.ve 


\section{The Execution of the Amplified Mother-Infant Program in Zulia State and the Attitude of the Beneficiaries}

\section{Abstract}

Two aspects which are not usually included in the majority of social compensatory policy programs were analyzed, taking as the case in study, the dynamic Amplified Mother-Infant Program (PAMI) in Zulia State (Venezuela). These aspects were: 1) an analysis of the process of program execution and 2) the attitude of the beneficiaries. The first element was studied using the following variables: organizational structure; operational strategies; information and publicity systems; supervision, control and follow-up mechanisms; geographic and social coverage; human, physical and financial resources; and efficiency in the fulfillment of goals. Attitudes were studied in terms of the level of knowledge and information on the part of the beneficiaries; opinions as to the impact of the program on their living conditions; their most frequent reaction when faced with partial or definitive changes in the programs; and their manner of participation in the programs. The basic objective was to insist on the need to integrate the design and evaluation of these aspects in programs to aid the poor, incorporating these aspects whenever they are the direct or indirect cause of the possible low impact of such policies.

Key words: Social compensatory programs, execution process, attitudes of beneficiaries, PAMI, Zulia, Venezuela.

\section{Introducción}

Este trabajo se ocupa de presentar la realidad del PAMI en el Zulia, evidenciando los obstáculos y errores de su proceso de ejecución, y caracterizando el tipo de actitud de sus beneficiarios, a fin de llamar la atención sobre: 1) la influencia de ambos aspectos en la escasa efectividad social del mismo; 2) la necesidad de evaluarlos bajo el enfoque del impacto social, y 3) la pertinencia de incluir esa evaluación en el diseño de los programas compensatorios.

Se parte de la idea que, aun con su sesgo compensatorio y pese a las insuficiencias del diseño, programas como el PAMI podrian tener mejores resultados si: 1) se previnieran y/o atacaran oportunamente los problemas que se presentan en su ejecución, especialmente lo relacionado con el componente educativo-formativo, y 2) si su implementación estuviese motivada más por el logro de los objetivos sociales, en términos de efectividad, y menos en términos de eficiencia-eficacia administrativa y politica.

En efecto, la intervención del Estado venezolano en el sector social, mediante programas compensatorios para atender la pobreza crítica y extrema, ha sido eficaz desde la pers- 
pectiva de la conservación de la "paz social", de la estabilidad y legitimidad del régimen, dado su carácter populista-clientelar. Sin embargo, desde el punto de vista de su contribución al bienestar social de la población ha sido inefectiva, en tanto asistencialista, incapaz in. cluso de mantener estacionarios los indicadores, bastante criticos de por si.

Por lo tanto, es necesario efectuar una revisión a fondo de los retrocesos que en materia social se han tenido, de los factores del empobrecimiento generalizado de la población y de los resultados de las modalidades de intervención estatal. Ello exige indagar en los factores explicativos, condicionantes y/o determinantes de los resultados de los progra. mas sociales.

Dentro de estos factores, en la mayoria de las investigaciones sobre el tema, se han sosiayado fundamentalmente los de carácter cualitativo (opiniones, actitudes, valores, expectativas de los beneficiarios y de los funcionarios involucrados), y lo atinente a las contingencias del proceso real de ejecución de los programas, que ponen de manifiesto proble. mas de coherencia interna del diseño y de coordinación o gerencia, entre otros.

Por la importancia estratégica de tales aspectos, en el presente articulo se revisa el proceso de ejecución y la actitud de los beneficiarios, de una de las principales estrategias de la política social compensatoria (el Programa Ampliado Materno Infantil, PAMI). Se trata de un resumen de los principales resultados de una investigación más extensa (Alvarado, 1997a), en la cual se analizan dichos elementos con referencia al funcionamiento del programa en el Estado Zulia desde 1990.

Como estrategia metodológica el estudio se nutre de información documental-estadistica, parte de ella extraida de las instituciones que a nivel central y en el Zulia han venido gerenciando el programa; asi como en fuentes primarias representadas por entrevistas semiestructuradas realizadas a funcionarios clave de ambos ámbitos (coordinadores y ejecutores directos del programa), y en encuestas a los beneficiarios de las comunidades seleccionadas, representados porlas madres de los niños destinatarios del programa.

Es importante acotar también que se tiene claro que para indagar aspectos cualitativos como actitudes, percepciones, valoraciones, no es la metodologia cuantitativa tradicional, con entrevistas de preguntas precodificadas y cerradas, la más pertinente. Sin embargo, parte de la información se recogió con estos instrumentos por cuanto se trata de un trabajo exploratorio, de mera aproximación a esa arista del problema, y principalmente porque el estudio marco del cual deriva, no tuvo como objetivo prioritario (sino complementario), evaluar esos aspectos cognitivo-conductuales.

\section{Caracterización General Formal del PAMI}

Como programa social compensatorio proplamente dicho, el PAMI se inicia en 1989 como parte integrante del Plan de Entrentamiento a la Pobreza y de la Estrategia de Atención Primaria en Salud, que privilegia las acciones hacia la comunidad antes que la atención estrictamente individualizada. La población objetivo de este programa son las madres embarazadas/lactantes y los niños menores de seis (6) años, en situación de pobreza crítica y/o extrema.

El objetivo general del PAMI como estrategia, es mejorar la situación de salud-nutrición de la población materno-infantil reduciendo los indices de morbimotalidad de esos grupos; siendo sus objetivos específicos, los siguientes: a. aumentar la cobertura médico-asistencial de la población materno-infantil, jerarquizada por criterios de riesgo, b. ampliar la atención a la madre y al niño mediante la provisión de productos lácteos, harina de maíz y polivitaminicos (estos últimos nunca se han suministrado), $c$. reforzar la Atención Primaria en Salud mediante acciones de educación, promoción y organización comunitaria en salud (MSAS, 1994).

Las funciones, actividades y objetivos de la Estrategia PAMI se desarrollan mediante 
tres (3) componentes: 1. Programa Atención en Salud (PAS), 2. Programa Alimentario Materno-Intantll (pami), y 3. Programa Educación y Promoción en Salud (PEC).

EI PAS se dirige a brindar servicios de naturaleza preventiva y curativa de tipo médi$\mathrm{Co}$, odontológico y nutricional, a través de las unidades de producción definidas como Atención Prenatal, Atención Postnatal, Atención al Infante y Atención al Preescolar; de la red de ambulatorios establecidos por el programa.

El componente alimentario consiste en la transferencla de leche en polvo completa y lactovisoy a las mujeres embarazadas y lactantes y a los niños entre seis (6) meses y seis (6) años, en los ambulatorios adscritos al programa.

El beneficio se suministra según grupo de riesgo socioeconómico y biológico, mediante una codificación que clasifica a la población objetivo de la siguiente manera: Prenatal: código 1 (2 kgs de lactovisoy) -el código 2 fue eliminado-; Postnatal: Código 3 ( 2 kgs de leche mensuales); Infantil Bajo Riesgo: Código 4 (2 kgs de leche mensuales); Infantil Alto Riesgo: Código 5 (3 kgs de leche mensuales); y Preescolar: Código 6 (3 $\mathrm{kgs}$ de lactovisoy mensuales).

Finalmente el componente Educación Comunitaria en Salus (PEC), prevé un conjunto de actividades educativas dirigidas a escolares, padres, representantes y grupos comunitarios, relacionados con conocimientos y prácticas de salud en las áreas materno-infantil, planificación tamiliar, sexualidad, oncología, salud mental, salud oral y otras. Procura la organización y participación de la comunidad en función del fortalecimiento de la atención primaria en salud, a través de investigaciones, actividades de formación y capacitación del personal, en salud y comunitario.

Los principales rasgos administrativos a nivel normativo son:

1) Los servicios del PAMI se prestan a través de la red de establecimientos de salud del pais, especiaimente la red de ambulatorios Urbano I, Rural II y ambulatorios adscritos a Hospitales, a través del personal médico, de enfermeria, nutrición, promoción social, capacitación y almacenamlento, directivos y supervisores de los organismos públicos, las $O N G$ involucradas y los medios de comunicación. La ejecución del PAMl está a cargo del Ministerio de Sanidad y Asistencia Social (MSAS), a través de la Dirección Materno-Infantil y demás dependencias involucradas en la Atención Primaria en Salud para la población objetivo; la Fundación Programa Alimentario Materno Infantil (Fundación PAMI) y el Ministerio de la Familia. A este último le correspondería el desarrollo del componente PEC.

2. Espacialmente el PAMI está organizado en tres niveles según componentes. El PAS está conformado de la siguiente manera: a) Nacional: Dirección General Sectorial de Salud y sus Direcciones Materno Infantil, Oncología, Epidemiología y Programas de Salud: b) Regional: Direcciones Regionales del Sistema Regional de Salud; y c) Distrital: Direcciones de los Distritos Sanitarios y las Coordinaclones de los Centros de Salud del PAS está prevista para desarrollarse, normativa-mente, mediante una serie de actividades que tienen como responsables equipos de los niveles central, regional, distrital y el personal operativo de la red de ambulatorios.

3. En cuanto al componente alimentario, en términos de coordinación está bajo la responsabilidad principal de la Fundación PAMI, cuya Junta Directiva está integrada por el presidente de la Fundación y representantes del MSAS, Ministerio de Educación, Ministerio de la Familia, CORDIPLAN (el organismo central de Planificación ) y el Instituto Nacional de Nutrición (INN).

La estructura organizativa para la ejecución está también integrada por tres niveles jerárquicos: nacional, regional y local (almacenes); y la estrategia operativa se desarrolla en dos fases: 1. Fase de Implantación (o de arranque del programa) y 2. la Fase de Ejecución propiamente dicha, que incluye: el proceso de distribución del producto desde el proveedor hasta el almacén; el proceso de supervisión y control, y el proceso de entrega a los beneficia- 
rios. Cada una de las fases comprenden actividades especificas, con sus respectivos responsables.

4. EI PEC también está organizado en tres niveles: central, regional y distrital. Al nivel central le corresponde: elaborar lineamientos, normas y procedimientos del programa; la coordinación interinstitucional; establecimiento de convenios y contratos para la ejecución del programa. Al nivel regional le compete elaborar el plan regional de salud y realizar actividades de control, supervisión y evaluación de lineamientos, normas y procedimientos del nivel central. El nivel distrital se encargaria de las funciones totalmente operativas, de ejecutar los programas de salud a través de los ambulatorios y en las comunidades donde éstos se localizan.

El nivel central lo integran las Direcciones: General Sectorial de Salud, Materno-Infantil y Promoción Social de la Salud del MSAS; y las Direcciones: General Sectorial de Coordinación Técnica del Programa de Educación Comunitaria, del Ministerio de la Familia. El nivel regional está representado por las Direcciones Regionales del Sistema Nacional de Salud del MSAS y del Ministerio de la Familia, y el nivel distrital, por las Direcciones de los Distritos Sanitarios y Coordinaciones de los centros de salud del MSAS y ONG de base comunitaria. La Estrategia Operativa del PEC implica una serie de actividades secuenciales donde participan todos los actores de su estructura organizativa.

\section{El Proceso de Ejecución de la Estrategia PAMI}

\subsection{La Ausencia de evaluación dél proceso de ejecución en el diseño del programa}

Es sabido que los problemas de las politicas públicas derivan no sólo de ejecución de éstas, sino también de su contenido, de las características de su diseño, que a su vez ocasionan obstáculos al suministro eficaz, eficiente y efectivo de los bienes y servicios que involucran. Esto es particularmente válido para las polfticas y programas sociales.

Entre las limitaciones más frecuentes, que se presentan como características comunes a casi todos los programas sociales, pueden mencionarse las siguientes: problemas derivados de la subordinación de la política social a la política económica y de la autosubordinación de la política social; problemas de enfoque, emanados de los modelos de poiltica social adoptados; falta de direccionalidad y objetivos claros; objetivos a largo plazo: gastos (altos costos); escalas (público masivo); complejidad administrativa por la existencia de una burocracia masiva; diferentes perspectivas de los funcionarios para asumir el programa; ausencia de seguimiento y evaluación ${ }^{1}$.

Sobre este último aspecto del seguimiento y especialmente de la evaluación de la ejecución, por su relevancia, es necesario detenerse un poco. En el diseño de los programas sociales no se preestablecen mecanismos concretos en esta materia, amén cuando se trata de iniciativas que cuentan con financlamiento de la banca multilateral. Generalmente se confunden el seguimiento y la evaluación con control; contraloria y "seguiduría", en términos financieros (destino de los recursos) con criterios sólo de eficacia, relacionados con el logro de metas cuantitativas ${ }^{2}$. De esto el PAMI, cofinanciado por el Banco Mundial, es un buen ejemplo.

Se excluye del diseño (y tampoco se ejecuta en la práctica) la evaluación de proceso y de impacto social de los programas, con criterios de efectividad social, lo cual no se compagina con los intereses y fines políticos que movilizan a los programas sociales. Pero 
también en gran medida hay un problema de ignorancia respecto a la importancia de la evaluación, y limitación en la formación o especialización de recursos humanos en la materia. Tal como lo expone Franco (1992:25) "...se ig. nora el desempeño de los programas y, sobre todo, el impacto que producen sobre la población objetivo. Se desconoce si sirven o no sirven; si sirven, cuánto sirven y el grado en que justifican su costo; y por último, a quiénes sirven y en qué medida, o dicho de otra manera, quiénes son los verdaderos beneficiarios. Si los programas no se evaluan se mantiene la inercia, se sigue haciendo lo mismo que en el pasado en la creencia que está bien, aunque no se sepa en realidad si lo está. Tampoco se sabe si podria haber una manera mejor de gastar los recursos y se desconoce, asimismo, si los recursos que se están destinando a esos programas están generando algún impacto sobre la población a la que se quiere atender".

La única salida a esta situación es evaluar la ejecución y su impacto, sin embargo, a pesar de las advertencias de los expertos al respecto, todavia son endebles las iniclativas en esta dirección. Sulbrandt (1993:310) lo expresa sin ambages: "... la mayoria de las politicas y programas sociales no recibe ninguna evaluación y en los casos en que ésta se ha aplicado se ha llevado a efecto de una manera que no satisface plenamente los requisitos de una investigación profesional. En esos pocos casos su objetivo ha sido tratar de presentar una comparación de las metas propuestas para los programas con lo realmente alcanza- do, tratando de dar una explicación ad-hoc sobre las discrepancias encontradas. En esos trabajos no se ha puesto el énfasis en los resultados e impactos de los programas sino en la carga de trabajo y en el número de actividades realizadas".

Ante el apremio de la crisis y la mengua del gasto social por las medidas de ajuste, pareciera que se ha comenzado a entender la necesidad de evaluar. Sin embargo, aún prevalecen los entoques y métodos tradicionales que sólo buscan conocer el grado de cumplim miento de las metas y explicar "ex-post facto" las causas del "desvio" de lo ejecutado respecto a lo formulado, comparando, con números y porcentajes, lo esperado y lo logrado. Como se demostrará más adelante, éste es el tipo de evaluación de la ejecución que priva en el PAMI.

\subsection{Hallazgos respecto del Proceso de Ejecución}

\section{a) Sobre la Estrategia General:}

Pese a que el PAMI se concibió como una estrategia de intervención social en el área de la salud y de la nutrición, de carácter sistémico e integral, el análisis de su funcionamiento revela un desfase en la ejecución de sus componentes o subprogramas, que se desarrollan como compartimientos estancos, transgrediendo así el diseño original y poniendo en cuestión el pretendido carácter de estrategia social integral. Presenta, por tanto,

2 Estos términos se entienden aquí de la siguiente manera: eficacia: grado de cumplimiento de los objetivos previstos, traducidos en metas, en el tiempo esperado. Eficiencia: optimización en el uso de los recursos destinados al logro de dichos objetivos. Efectividad o impacto social: nivel de satisfacción en la población objetivo de la necesidad que el programa tiene por objeto atender. Expresa la relación entre los resultados/efectos logrados y los objetivos deseados. Da cuenta de los cambios ocurridos en el periodo considerado, que pueden atribuirse al programa en cuestión. 
serios problemas de articulación, coordinación o gerencia.

Aun cuando, según el diseño, el PAMI se orienta a generar fundamentalmente un impacto estructural en la población materno infantil en situación de pobreza extrema; en la práctica se comporta como un programa de corte netamente compensatorio, que ha desviado su objetivo central de fortalecer la Atención Primaria en Salud con énfasis en la prevención.

Esto se demuestra en el hecho de que aproximadamente el $90 \%$ de sus recursos presupuestarios se asignan al componente allmentario, que privilegia el reparto de leche y de lactovisoy iplanteado normalivamente como un "anzuelo táctico" para atraer al beneficiario a la consulta preventiva), antes que la asistencia médica. Otra evidencia es que el Programa de Educación Comunitaria en Salud (PEC) no fue ni es desarrollado en igualdad de condiciones a los otros subprogramas, aun cuando el componente educativo es el más importante y el que podria aproximar hacia el éxito a todo programa social.

Relacionado con esto, hay un marcado interés en la Fundación PAMI y en el MSAS por lograr eficacia y eficiencia, medidas en función de la cantidad de producto entregado y del número de beneficiarios activos en los servicios del Programa de Atención en Salud (PAS). No así en lograr efectividad o impacto social, en las condiciones de vida de la población destinataria, aspecto que se desconoce pues para ello no existe un sistema de seguimiento y evaluación de la Estrategia.

\section{b) Sobre la Estructura Organizativa y la Estrategia Operativa}

La coordinación, red de relaciones y de rendición de cuentas sólo está definida y se ejecuta al interior de cada componente, no intercomponentes. No hay claridad de competencias y df articulación entre las instituciones Involucradas, to que afecta la planificación y control sobre la marcha global de la estrategia, habida cuenta además de las divergencias en los registros que llevan cada una de aquéllas. Esto también contribuye a la dispersión de esfuerzos, al incremento de costos y hace más difícil el seguimiento, control y evaluación.

Los bienes y servicios que incluye la Estrategia se suministran desfasadamente por los problemas de articulación y de visión de conjunto. Se prioriza el subprograma alimentario (que está altamente centralizado en la Fundación PAMI-Caracas), sobre el PAS ; los ambulatorios no cuentan con todos los servicios incluidos en este componente, los usuarios están poco informados de los que existen, y no se ejecuta el PEC.

En esto último todos los entes salvan su responsabilidad, especialmente el Ministerio de la Familia, a quien por definición y por diseño, le correspondería desarrollario, asi como lo hace para otros programas sociales, como por ejemplo, los Hogares de Cuidado Diario y los Multihogares.

El suministro de productos lácteos, aun cuando es lo que se realiza con mayor controly regularidad, se ha visto afectado en los ambulatorios del Zulia por frecuente ruptura de stock (a causa de la centralización del programa) y por los paros médicos. Ello ha incidido en los casos de deserción de beneficiarios y en la disminución de su asistencia al servicio médico, porque la obtención del producto sigue siendo lo más atractivo para ellos.

Los paros del personal de salud inciden directamente en ambos aspectos por cuanto para acceder al producto se debe pasar primero por la consulta reglamentaria. Esto se ha convertido en un círculo vicioso que genera necesidad de reprogramar constantemente $y$ ocasiona a los beneficiarios gastos de transporte innecesarios al ambulatorio, acumulación de productos en el almacén con riesgo de deterioro (en el caso del Zulia este riesgo es mayor a causa del calor) y largas colas de beneficiarios en el almacén para retirar el producto, cuando el suministro se reanuda.

La no correspondencia de horarios entre la atención médica y el almacén, la insufi- 
ciencia de personal médico especializado, de material médico quirúrgico y de equipos de apoyo, la frecuente falta de papelería, aumentan el tiempo para la obtención de una cita y disminuye el registro de nuevos beneficiarios.

\section{c) Sobre los Sistemas de Informa- ción y Mecanismos de Difusión}

No hay un sistema único, homogéneo y sistematizado de información sobre la ejecución de la Estrategia. Cada componente lleva sus propias estadisticas en algunos casos, incluso con resistencia del personal (caso de los empleados del MSAS). La Fundación PAMI, a través de la Oticina Zulia, sílleva al día sus re. gistros, pero al no estar compatibilizados con los del MSAS, se díficulta el análisis del proceso, de su eficacia y efectividad.

Además, las estadísticas se llevan manualmente y caso por caso (una ficha por beneficiario), lo cual impide una visión de conjunto. Sólo la Oficina Zulia dispone de un computador donde se transcribe la información que llega de los ambulatorios y se reporta a Caracas. Es decir, no hay un flujo de información por red ni de los aimacenes a la Oticina Zulia ni de ésta hacia la Fundación Central (esto es un proyecto desde fines de 1995).

Respecto de la difusión hay bastantes fallas: la principal es el incumplimiento del PEC tal como estaba previsto inicialmente. La Fundación PAMI pretendió sustituir a los promotores sociales por los almacenistas dejándoles a éstos la actividad de información y promoción, lo cual ha fracasado porque es muy dificil para ellos atender el almacén (con todas las contingencias del proceso de entrega de producto) y al mismo tiempo "educar" a los beneficiarios.

Por otra parte, aun cuando los ambulatorios disponen del servicio de Trabajo Social, éste no tiene injerencia en las actividades de orientación y promoción. Asimismo ocurre con el servicio de nutrición. Al respecto el MSAS argumenta falta de recursos para pagar más personal. Por ello, lo poco que se hace en este sentido depende de la iniciativa y buena voluntad de los Médicos-PAMl y de las enfermeras.

Los medios de Intormación y difusión masiva (prensa, radio y televisión) no son utilizados por la Fundación PAMI y por el MSAS para la promoción de la Estrategia. La Fundación edita mensualmente un boletin informativo, pero éste no llega masivamente a los beneficlarios reales ni a la población objetivo potencial. Todo ello explica los problemas de desconocimiento y desinformación que existen entre los beneficiarios y explica también por qué a la mayoría de éstos sólo les interesa acceder a los productos lácteos.

\section{d) Sobre la Supervisión, Control y} Seguimiento

Por causa de los problemas de información y de la no previsión en el diseño de metodologías integradas de control y seguimiento, la Estrategia presenta dificultades en este sentido lo cual, a su vez, dificulta realizar evaluaciones de metas, proceso e impacto. Lo que cada institución corresponsable del PAMI entiende por supervisión, control y seguimiento, lo realiza respecto de las actividades que cada componente desarrolla (no hay un sistema homogéneo).

Por ello hay problemas para contabilizar la población atendida, por primera vez y sucesivamente, tanto por el PAS como por el subprograma alimentario; y también para revisar periódicamente el cumplimiento de objetivos y/o metas. Aqui también se observa que privan los criterios de eficacia y de eficiencia, mas no de efectividad: racionalización de recursos, criterios de mercado en la administración de los servicios, etc. (esto es claro en la Fundación PAMI), obviando que la eficacia y la eficiencia, más que fines en sí mismos, deberían ser medios para alcanzar el objetivo de mejorar las condiciones de salud y nutrición en la población de reterencia.

Ello explica el empeño de la Fundación PAMI en la supervisión del personal y control de actividades, que se traducen en seguimien- 
to numérico sin considerar el impacto social: número de beneficiarios atendidos, cantidad de producto entregado, número de consultas efectuadas, número de almacenes abiertos, número de ambulatorios adscritos, etc. Pero no, por ejemplo, cuántos casos de desnutrición se han recuperado, cuántas muertes intantiles y maternas se han evitado, etc. Se entiende to primero y no lo segundo como indicador de éxito del programa, por lo cual hay de fondo un problema de conceptos.

\section{Social}

\section{e) Sobre la Cobertura Geográfica y}

A pesar de los esfuerzos de la Fundación PAMI y del MSAS, y de los elevados indices de desnutrición, mortalidad materna e infantil existentes en el Zulia, la cobertura en 1995 apenas alcanzaba al $50 \%$ de los ambulatorios en el caso del componente alimentario y a $33 \%$ en el caso del PAS, según informaron los respectivos coordinadores. En ello ha incidido la masificación del PAMI en todo el país (por lo cual las demandas son múltiples y permanentes) y la centralización del mismo, en la región no hay autonomia para establecer metas ni criterios de selección y focalización.

Relacionado con esto, como el criterio básico de acceso al programa es la autoselección de los beneficiarios, personas no estrictamente pobres se han incorporado al programa sin que haya controles al respecto, razón por la cual ha habido incrementos consecutivos en todos los códigos-PAMI, excepto a partir de 1995, dada la sustitución de leche por lactovisoy en algunos de ellos. Sin embargo, la permanencia en el programa (beneficiarios sucesivos) presenta dificultades por las contingencias que afectan el suministro de los bienes y servicios del PAMI.

f) Sobre los Recursos Humanos, Fisi. cos y Financieros

Hay insuficiencia de personal activo incorporado a la Estrategia, no son empleados fj- jos y su dedicación al PAMI es de medio tiempo, en el caso del personal del MSAS. El personal que depende de la Fundación PAMI también es escaso (por ejemplo, hay un solo almacenista por cada ambulatorio). Ello ocasiona saturación de actividades en el personal activo: las tareas del PAS recaen en el Médico-PAMI y en las enfermeras; que se ven abrumadas por la papelería que deben llevar, presentán. dose frecuentes quejas al respecto.

En cuanto a los recursos físicos, los ambulatorios presentan problemas de infraestructura (reducido espacio de las salas de espera y de los almacenes, insuficiencia de consultorios, etc.) y también de dotación: el material médico-quirúrgico es prácticamente nulo, por lo cual las actividades del PAS se realizan muy limitadamente, dentro de los pocos servicios activos.

Respecto del presupuesto, el principal sesgo es que las decisionns con él relacionadas están sumamente centralizadas. La Dirección Regional de Salud (y con ella la coordinación del PAS) y la Oficina Zulia de la Fundación PAMI, no tienen autonomia para decidir ni en la más pequeña actividad que implique erogaciones financieras.

Sólo pueden estimar las necesidades de recursos a partir de las cuales las dependencias centrales calculan el presupuesto para la región. Generalmente, no hay correspondencia entre el presupuesto solicitado, el asignado y el ejecutado; $y$ en general, el presupuesto del PAMI en el Zulia (caso del componente alimentario) en el período 1990-1995 apenas alcanza al $3 \%$ del presupuesto total de la Estrategia.

\section{g) Sobre la Eficacia o Cumplimiento de Metas de la Estrategia}

Por los inconvenientes de planificación, : Macion, stema de intormación, etc., la Estrategia PAMI ha venido marchando prácticamente a la deriva, por cuanto no se estiman anualmente metas concretas ni en relación al número de beneficiarios que se incorporarian, 
ni al número de consultas preventivas que se realizarán y a la cantidad de producto que se entregará anuaimente.

En los años iniciales se establecieron metas globales, no coincidentes entre el MSAS, la Fundación PAMl e incluso el Banco Mundial (quien cofinancla el programa), las cuales resultaron sobreestimadas por lo que no se alcanzaron. Pero luego, la meta fue sustituida por buenos propósitos generales ${ }^{3}$. Esto hace que la eficacia del componente alimentario y del PAS se mida en términos generales: por el volumen de beneficiarios, de producto entregado y consultas realizadas, pero no en relación a un porcentaje de meta cumplida anualmente.

\section{La Actitud de los Beneficiarios del PAMI}

\subsection{Consideraciones teóricas}

La perspectiva de "arriba hacla abajo" según la cual se formulan los programas y servicios sociales, ignora totalmente la participación, percepción y conductas reales (esto es, la actitud) de los usuarios. Se obvia la existencia de múltiples situaciones relacionadas con el beneficiario directo, no sólo desde la pers. pectiva macrosocial, en tanto sector de población vulnerable y receptor anónimo-pasivo, sino como destinatario último de la acción gerencial en una concepción micro-operativa.

Esto pasaría por entender que el logro de los magnos objetivos de los programas sociales "... no se limita ni reduce al replanteamiento de las politicas sociales, al incremento significativo de los recursos asignados y a la transformación de la dimensión institucional; se trata de algo más que gerenciar programas y niveles organizacionales. Muy por el contrario, se ubica en superar la pobreza y ello exige un proceso de dignificación de quienes la pa* decen y su materialización en una elevación real de su nivel de vida $(.$. ). De lo anterior se deriva que no es posible participar en la conformación y ejecución del proyecto social que mejor contribuya a la realización de los objetivos (...) si se desconoce la cotidianidad del beneficiario ..." (FONSECA, 1997:12).

Dentro de esa "cotidianidad del beneficiario" se hace necesario conocer e incorporar en el diseño de los programas sociales lo referente a la actitud, como factor de potencial influencia en la efectividad social de aquéllos; y entender que no necesariamente ha de existir o existe un solo tipo de beneficiario (el receptor-pasivo), sino que también podría hablarse de una diversidad en este sentido, como plantea Ahumada (1996) en la reseña que se introduce en líneas posteriores.

No existiendo tampoco un concepto único sobre actitud ${ }^{4}$, es conveniente aclarar cómo se entiende a lo largo de este trabajo. Rodríguez (1983:331-332), basándose en diez definiciones presentadas en distintos manuales de psicologia social, sintetiza los siguientes elementos, esenciaimente característicos de las actitudes sociales, en torno a los cuales articula una definición; "a) la organización duradera de creencias y cogniciones en general; b) la carga afectiva en favoro en contra; c) la predisposición de la acción; d) la dirección a un objeto social. Por tanto, podemos definir la actitud social como una organización duradera de creencias y cogniciones en general, dotada de una carga afectiva en favor o en contra de un objeto social definido que predispone a una acción cohe(1994).

4 Segũn Rodriguez (1983), ya desde 1935 Aliport recopiló cien definiciones. 
rente con las cogniciones y afectos relativos a dicho objeto".

Segün dicho autor (1983:331-333) las actitudes sociales están compuestas por tres elementos claramente discemibles: un componente cognoscitivo, un componente afectivo y un componente conductual: "Para que exis" ta una actitud es necesario que exista también alguna representación cognoscitiva de dicho objeto $(. .),. \ldots$ el componente afectivo, definido como el sentimiento a favor o en contra de un determinado objeto social, es realmente característico de las actitudes sociales(...). Finatmente la posición generalmente aceptada por los psicólogos sociales es aquella según la cual las actitudes poseen un componente activo, instigador de conductas coherentes con las cogniciones y los afectos relativos a los objetos actitudinales ...".

Tomando en cuenta esta triada de componentes (lo cognitivo, lo afectivo y lo conductual) en esta investigación se entiende como actitud un concepto ampliado que incluye los siguientes elementos: 1) el nivel de información y/o conocimiento de los beneficiarios sobre el programa; 2) su opinión o apreciación sobre la pertinencia, el proceso de ejecución, la eficacia y la efectividad del mismo; 3) su reacción y/o expectativas ante un posible cambio del programa y 4) las formas de participaciơn en éste.

Estas subdimensiones a su vez pueden resumirse, siguiendo a Ahumada (1996) en dos grandes aspectos: la percepción y las prácticas cotidianas asumidas por los beneficiarios en el área de los programas y servicios sociales, entendido como un sistema de relaciones que incluye usuarios, prestadores, autoridades y funcionarios públicos que contribuyen para establecer las condiciones especificas en que éstas se producen.

El concepto de "campo", introducido por Pierre Bordieu (Ahumada, 1996:6-8), constituye un recurso analítico que permite entender estos aspectos más cualitativos de las politicas sociales. Se entiende por campo a "espacios de juego históricamente constituidos", en los cuales cada actor ocupa determinadas posiciones.

Aquellos que hegemonizan el capital especifico, que es el fundamento del poder o de la autoridad concreta característica de un campo, se inclinan hacia estrategias de conservación, es decir, posiciones que dentro de los campos de producción de servicios tienden a defender la ortodoxia, mientras que los que disponen de menos capital (por ejemplo, los prestadores municipales) se inclinarían a emplear estrategias de transformación, entendido por el mencionado autor como "herejia", es decir, como ruptura generaimente ligada a la crisis.

Del lado de los beneficiarios la estrategia básica sería la demanda de bienes y servicios sociales, en tomo de lo cual podría o no estructurarse relaciones de tensión con las prácticas conservadoras del Estado, o "herejes" de otros actores, dependiendo del tipo de usuarios y de consumo, es decir, de la modalidad de la demanda.

En este sentido, Ahumada (1996:15. 16), relacionados con la demanda de servicios de salud en Argentina, distingue tres tipos principales de beneficiarios o usuarios y de consumo: "Usuarios conformistas. Estrategias : se acostumbran a recibir cualquier tipo de trato y servicio; aceptan todo; cuando tienen problemas se callan; tienen temores; para ellos todo funciona blen; dependen del dispensario en cuestiones básicas como la leche y los bolsones; valoran el uso de hierbas medicinales. Los usuarios conformistas tienden a adoptar "estrategias de conservación", como unintento de lograr minimas seguridades (la entrega de la leche, la atención médica básica) en una realidad que no les presenta casi ninguna alternativa. Existen en estos grupos criterios pragmáticos para enfrentar la vida cotidiana. La no asistencia a reuniones, la no capacitación en prevención podría estar ligado a la consideración de que de tales actividades no es posible extraer una utilidad inmediata. En la vida 'atenida a las urgencias' dificilmente se pueden llevar adelante prácticas cuyo rédito 
no es percibido de manera inmediata. Los intereses en juego se expresan en la necesidad de accesibilidad a los servicios de salud. Los usuarios conformistas no acceden a los prestadores ortodoxos y si bien aceptan a los prestadores herejes se encuentran en una situación de contradicción con ellos.

Usuarios activos. Estrategias : los que buscan, van de un lado a otro; los que van siempre; los que se organizan y se quejan; los que tienen una actitud activa en relación a los servicios de salud, no sólo de recibir sino de buscar alternativas mejores. Se sienten con derecho a recibir un buen trato. Valoran la salud. Los usuarios activos se sienten con derecho a reclamar la prestación de los ortodoxos y se encuentran en franco enfrentamiento con los herejes.

No usuarios: automedicación y prácticas populares (hierbas medicinales). No participan de las leyes del campo; los que no concurren más (deserción); no aceptan control (desnutridos, embarazadas adolescentes). Concurren para algunos servicios (niños) y escasamente para adultos".

Al tomar como referencia las anteriores consideraciones teóricas, corresponde ahora analizar los resultados de la encuesta aplicada a las madres beneficiarias del PAMI de las zonas de influencia de los ambulatorios del Estado Zulia, en torno de las cuatro (4) subdimensiones identificadas dentro de la categoria o dimensión "actitud de los beneficiarios" y, a partir de esa información, caracterizar al usuario típico de la estrategia PAMI en el Zulia.

\subsection{Hallazgos respecto de la Actitud de los Beneficiarios}

1) Hay un marcado desconocimiento sobre qué es exactamente elPAMI, cuáles son sus objetivos y los servicios/beneficios que ofrece cada uno de los subprogramas. Lo único claro es el reparto de productos lácteos y que para acceder a ellos obligatoriamente hay que pasar por una consulta. El fin se convierte así, en el medio. La promoción, orientación en salud y nutrición dependen de lo poco que el Médico-PAMI, las enfermeras y el almacenista puedan hacer durante la consulta, es decir, no corresponde a una estrategia direccionada con fines educativos.

2) La percepción que la mayor parte de los beneficiarios entrevistados tiene del PAMI es que constituye una importante ayuda del gobierno para las familias pobres y hasta es visto por algunos como "el mejor programa social del Gobierno". Hay satisfacción con la cantidad y calidad del producto lácteo y con la atención médica recibida. Como limitaciones del proceso de ejecución señalan la frecuente falta de producto en el almacén, el limitado horario de consulta y los inconvenientes ocasionados por los paros médicos. Los pocos beneficiarios que expresaron conocer los objetivos del PAMI, opinan que si ha cumplido con éstos.

3) La forma generalizada de participación en el PAMI es: aprovechando el beneficio (es decir, retirando el producto y asistiendo a la consulta médica). No hay otra vinculación con el ambulatorio ni con las respectivas juntas de vecinos para coadyuvar con el programa.

En general, las comunidades presentan escaso desarrollo organizativo, y no demandan colectivamente mejoras en aquél, ni siquiera cuando se suspende el servicio. La única participación de la junta comunal es colaborando ocasionalmente con la Fundación PAMI en el traslado de los productos al almacén. Más que estrategias de participación, los beneficiarios desarrollan estrategias de consumo y de conservación.

4) La reacción más extendida de los beneficiarios hacia algunos cambios parciales introducidos al programa (por ejemplo sustitución de la leche por el lactovisoy en algunos códigos) es de rechazo. Asimismo, hay total desacuerdo con el posible reemplazo del programa por otro y más aún con una definitiva eliminación del mismo.

5) Finalmente cabe acotar que la ratificación del PAMI en la Agenda Venezuela, jun- 
to al resto de programas compensatorios asistenciales focalizados, no parece modificar la actitud reseñada anteriormente, en las distintas subdimensiones en que metodológicamente se subdividió esta categoria.

En efecto, una encuesta de opinión sobre los programas sociales de la Agenda Venezuela, efectuada en la ciudad de Caracas por la Universidad Católica Andrés Bello y el Ministerio de la Familia (UCAB-MIN. FLIA), 1996:3-10), registró significativos hallazgos entre los cuales pueden resumirse, los siguientes:

- Baja información sobre los programas sociales y en general desconocimiento de la Agenda Venezuela y de sus políticas: el $55.37 \%$ ignora o es incapaz de mencionar uno de los programas, y el $70 \%$ tampoco sabe quién es el organismo responsable de la ejecución.

- La opinión sobre los programas sociales tiende a ser favorable y en general hay alta satisfacción con ellos.

- Correlación pobreza-valoración positiva: cuanto más pobre, más proclive se es a los programas sociales. Por ejemplo, del estrato E casi el $70 \%$ está "muy a favor" o "a favor".

Otro estudio de opinión más reciente, realizado también por la UCAB conjuntamente con la empresa "Barómetro" pués del sondeo anterior (El Universal, 11-04$98,1-4$ ), afirma que "se fortalece la opinión favorable a la Agenda Venezuela, en tanto que el porcentaje de población que está de acuerdo con ésta es muy cercano al de la que manifiesta desacuerdo ( $41 \%$ y $46 \%$ respectivamente). Según dicha encuesta el escepticismo tiende a reducirse cada año porque de $58 \%$ de personas que creian que la Agenda no tendría ningún impacto positivo en 1996, descendió a $34 \%$ en 1998 , es decir, aumentó la proporción que confia en el cumplimiento del Programa en un $24 \%$.

En 1998 se estaría ratificando la correlación estrato social-opinión favorable antes comentada, puesto que fueron las clases $D$ y $E$ quienes emitieron la mejor opinión sobre la aplicación del programa económico-social, al resultar con $42 \%$ de apoyo.

Tanto los hallazgos de la encuesta efectuada dentro de esta investigación como de los sondeos de la UCAB, evidencian un tipo de beneficiario conformista, según la tipología de Ahumada, totalmente pasivo-receptor y ratifican, una vez más, el nivel de internalización de la cultura clientelar populista en el venezolano, favorable a la reproducción de percepciones y prácticas como las encontradas, antes que a un cambio de actitudes.

\section{Incidencia del Proceso de Ejecución y de la Actitud de los Beneficiarios en el Impacto Social del PAMI}

En una investigación anterior a ésta (Alvarado et. al, 1996), se analizaron los impactos (indirectos) del $\mathrm{PAMI}^{6}$ en las condiciones de salud/nutrición de la población venezolana, en general, y algunos en la población de referencia de la Estrategia. En líneas generales se es. timó como bajo el impacto social, en ello confluyen múltiples factores, dos de los cuales son los considerados en este trabajo.

Este sondeo fue efectuado entre enero y marzo de 1998, en una muestra aleatoria simple estratificada por cuotas de 900 hogares, en el área metropolitana de Caracas y en el estado Miranda.

No fue posible estudiar los impactos directos dada la ausencia de seguimiento de indicadores de los efectos reales de los programas compensatorios en poblaciones-objetivo específicas. 
Cabe reiterar que aun cuando la Estrategia PAMI alcanza algunos logros relacionados con el número de beneficiarios incorporados, con la cantidad de producto entregado y con el incremento de las consultas preventivas, pues la preocupación está centrada en las repercusiones del programa en cuanto a su eficacia administrativa; ni siquiera a nivel del "deber ser" (diseño) se prevé la cuestión de la efectividad o impacto social, por lo cual no se realiza seguimiento ni evaluación de los efectos de la Estrategia en la situación nutricionaly de salud.

Esta cuestión resulta bastante dificultosa realizarla porque los formularios de registro de beneficiarios, además de los problemas de subregistro que presentan, no recogen la información pertinente para elio, y aun cuando así fuese, al registrarse las fichas mensualmente y beneficiario por beneficiario, es dificil seguirle la pista en las estadisticas a quienes, luego de ingresar en el código 1, permanecen en él como beneficiarios hasta egresar normalmente del programa.

Un ejercicio de este tipo, se intentó a manera de ensayo en los ambulatorios de la muestra respecto del estado nutricional de las madres del código 1, del peso al nacer de los niños del código 4 (hijos de madres sucesivas desde el código 1), y del estado nutricional de los niños del código 4 al ingresar a éste.

En general, en ambos ambulatorios la mayoria de las prenatales del código 1, que se mantienen hasta el código 4, presentan un estado nutricional normal y el número de éstas aumenta al egresar de dicho código. Aunque no son despreciables los casos que se presentan de déficit de peso y de sobrepeso, son mucho menores. El peso de los niños al nacer en su gran mayoria es mayor de 2.500 gramos (peso promedio normal) y llegan al código 4 con un estado nutricional normal, aunque se registran algunos casos de desnutrición leve y de sobrepeso.

Esta información general, no fidedigna totalmente, habla de algunas tendencias favorables de la Estrategia PAMI. Sin embargo, en ningún caso sustituye los desfavorables indicadores que se presentan en el Zulia ( $y$ en Venezuela) en salud y en nutrición (Alvarado et. al, 1996), que indirectamente señalarian bajos impactos sociales de la Estrategia PAMI, los cuales pudieran verse acentuados (tampoco es posible sostener conclusiones categóricas al respecto), por causa de las fallas y limitaciones detectadas en el funcionamiento o proceso real de la Estrategia.

En general el PAMI es de escasa pertinencia y direccionalidad ante el problema que busca atacar, por lo cual, de entrada no podrian esperarse de él altos impactos, desde el punto de vista de la efectividad social: la naturaleza y magnitud de las necesidades involucradas, en lo nutricional y en salud, lo hacen insuficiente como estrategia de intervención dada su orientación básicamente asistencialista.

No obstante, siendo optimistas, aún con el carácter compensatorio del programa, si se implementase según las funciones y procedimientos previstos en el diseño, o introduciendo durante la ejecución cambios parciales, se podrían esperar del PAMI algunos efectos lavorables, siempre y cuando hubiese más preocupación por el logro de los objetivos previstos.

El caso del programa compensatorio "Vaso de Vida", en el Estado Lara, parece ase* verar esto: simplemente garantizando que el producto llegue efectivamente al beneficiario, incorporando otros mecanismos y agentes de distribución, se estaría acercando a lo esperado del programa, a pesar de los vacíos e inconsistencias de su diseño (Bilbao y Pachano, 1998).

Lógicamente si se hacen confluir las dos cosas: los problemas internos del diseño y el desfase de la ejecución respecto de las funciones, actividades y responsabilidades normativas (dando lugar a diversas fallas e irregularidades), es menos probable que el PAMI genere las "resonancias" esperadas.

La tesis que se comparte aquí es que pese a sus deficiencias programáticas, en la práctica, los programas compensatorios, po- 
drian tener otros resultados si se mejorara su gerencia, especiaimente si se instrumentan los mecanismos de relacionamiento-articulación, comunicación y promoción del mismo en los niveles operativos que involucra (nacional, regional y local), y entre los distintos actores implicados, en base a estrategias de negociación y al cumplimiento de los mecanismos de control y supervisión establecidos.

Si además de que éstos no son idóneos, tampoco se cumplen, no es dificil entender la realidad del programa. De esta manera se tiene que el proceso de ejecución es una variable coadyuvante o limitante de la efectividad social de un programa, por las razones ante. riormente argumentadas.

Con respecto a la actitud de los beneficiarios (en los términos aqui entendida y estudiada) es uno de los condicionantes del impacto social del PAM!, por las siguientes razones:

1 - La percepción y prácticas o estrategias cotidianas de los beneficiarios en torno del programa: acomodaticias o adaptativas, no conllictivas sino conservadoras; nutre y recrea lo que se podría denominar el círculo vicioso de la política social venezolana: es decir, la permanencia en el tiempo, sin perspectivas de cambio a mediano y largo plazos, de los programas asistencialistas compensatorios, pese a que la realidad empirica y las estadísticas disponibles (incluso oficiales) les señalan bajos impactos.

2.- La actitud de los beneficiarios no es considerada por los formuladores y decisores públicos como una variable de importancia crucial en el éxito de los programas sociales, por lo cual se obvia totalmente en su disefio. De antemano, y de una vez y para siempre, se les asume como entes "dados", pasivos (y no se concibe la posibilidad de entenderlos de otra manera), por la verticalidad característica del sistema político venezolano, que es el contexto global de la política social.

Lo contrario generaria problemas de legitimidad (si los beneficiarios se tornaran "activos" o "herejes", en los términos de Bordieu) y se veria comprometida la eficacia de la política social, según los propósitos no declarados o "agenda oculta" de la misma, es decir, garantizar estabilidad o "paz social", en lo cual, como hemos señalado, ha resultado eficaz.

Aun cuando el Estado es reiterativo, en el discurso político y en la planificación oficial, en la importancia de la participación de la socledad civil, en general, y de la población destinataria de los programas focalizados, en particular ${ }^{7}$, se obvia que la participación está condicionada por la actitud, por lo cual es imposible generar cambios de conducta colectiva si antes no se interviene el aspecto cognitivo ${ }^{8}$ (qué y cómo piensa la población y por qué), es decir, sino no se promueve un cambio de actitudes en los beneficiarios, su rol pasivo-conformista se eternizará e imposibilitará una participación activa, crítica y autogestionaria respecto de los programas sociales.

Esto, a su vez, alimenta el círculo vicioso de la política social antes referido, por cuanto aunque el discurso sea otro, oficialmente aún se entiende por participación básicamente la oportunidad de que la población de referencia pueda acceder y usufructuar de los bienes y servicios sociales que asistencialmente provee el Estado, y es eso lo que principalmente se promueve, antes que una intervención for-

7 El Plan de Solidaridad Social, el IX Plan de la Nación y la Agenda Venezuela son ilustrativos al respecto (Véase: Alvarado et. al, 1996).

8 Un tercer elemento, de suma importancia, no analizado en este trabajo, es el componente emocional (qué sienten, cómo se sienten los beneficiarios en torno a los programas sociales), lo cual toca probiemas psicosociales como la autoestima colectiva (Bustos, 1998). 
mada, consciente, organizada y autogeneradora de los grupos-objetivo.

Por eso es que para los planificadores, decisores superiores y "gerentes" de programas sociales, es tan fácil: 1) responsabilizar al beneficiario de su escasa organización y participación en aquéllos, y 2) pensar que la mera convocatoria pública "a participar" que fre. cuentemente se realiza a las juntas de vecinos, asociaciones civiles, cooperativas, Iglesia, ONG, etc., es suficiente para que la "sociedad civil" aporte su "granito de arena" y participe, dentro de los parámetros del esquema estandarizado de la política social vigente en el país.

3. Finalmente, la actitud de los beneffciarios, caracterizada por la inmediatez $y$ emergencla ("ya", "ahora") con que procuran los bienes y servicios que suministran programas sociales como el PAMI, aleja a éste de su aspiración formal-normativa (prevista en el diseño) de convertirse, a largo plazo, en un programa más de corte estructural-promocional y de alto impacto social, que asistencialistacompensatorio.

En resumen, si desde el punto de vista cognitivo-afectivo y conductual no hay tendencias en el beneficiario a favor de un cambio en el programa, o hacia otras iniciativas de mayor pertinencia; es lógico suponer que este tipo de actitudes convergen en los resultados y efectos observados de programas compensatorios como el PAM!

\section{Conclusiones}

Con arreglo a las revisiones y argumentaciones efectuadas en esta investigación, por las caracteristicas que presentan tanto el proceso de ejecución del PAMI, como la actitud de sus beneficiarios, ambos aspectos ejercen influencia en el bajo impacto social de dicha Estrategia.

Otros factores condicionantes tocan problemas que tienen que ver con: 1) el enfoque de la Estrategia PAMI (su sesgo asistencialista y la restringida concepción de la salud y la nutrición a la cual responde), y 2) las inconsistencias in- ternas del diseño. Tanto en su formulación como en su ejecución, la salud se entiende como la mera ausencia de enfermedad y la nutrición como un asunto únicamente alimentario.

En el diseño es evidente la atención especial que recibe la distribución de productos lácteos, la ausencia de una evaluación ex-antes, cuantitativa y cualitativa, asi como también de las capacidades reales disponibles para la ejecución de la Estrategia. Asimismo, hay ausencia de metas concretas y de operaciones y acciones acordes con los objetivos formulados.

Un gran vacio existe igualmente respecto de las formas especificas de interrelación y coordinación entre los decisores y ejecutores de los distintos componentes, y en la distribución de responsabilidades funcionarias.

A pesar de la eficacia relativa de la Estrategia, todas estas limitaciones de diseño y de implementación, alejan al PAMI del propósito básico que se trazara en 1989, cuando pasa a sustituir $y$ absorber las funciones del Programa de Asistencia Alimentaria del Instituto $\mathrm{Na-}$ cional de Nutrición (INN), dirigido a mujeres embarazadas para convertirse formalmente en una estrategia estructural e integral, capaz de coadyuvar efectivamente en el enfrentamiento de problemáticas relacionadas con la salud y nutrición de la población matemo-infantil, en condiciones de pobreza extrema.

En general, la evaluación realizada por el Consejo Nacional para el Seguimiento, Supervisión y Evaluación de los Programas So. ciales (CONASSEPS) para el caso Venezuela (1994), y este estudio para el caso Zulia, son bastante ilustrativos de los nudos críticos que le restringen efectividad o impacto social al PAMI, todo lo cual reclama cursos altemativos de acción, que pasan por una profunda revisión de la política social vigente en el país, especialmente de los programas de enfrentamiento a la pobreza. Justifican, ante todo, una estrategia social alternativa, no sólo para el Estado Zulia sino para toda Venezuela, puesto que estas situaciones son comunes al resto de programas compensatorios. 
El análisis efectuado respecto de estos factores condicionantes del impacto social del PAMI, a menudo no tomados en cuenta por quienes diseñan y gestionan los programas sociales, remite a una conclusión central: hay de fondo al menos tres problemas fundamen. tales. Uno de enfoque e interpretación, otro de gerencia y un tercero de ignorancia.

El primero se pone de manifiesto en varios aspectos: en la interpretación simplista que los gobiernos realizan de la realidad social del país, amparados en la teoría del "derrame"; en los restringidos conceptos de pobreza, sa lud y nutrición que manejan; en la forma como se concibe al beneficiario o usuario (como un ente totalmente nulo, pasivo), y especialmente en la orientación asistencialista-compensatoria que nutre los programas sociales, aunque están dirigidos a problemas estructurales.

Ello le resta direccionalidad a la política social y la sume en un círculo vicioso, cuyo producto tangible es su incapacidad para detener el deterioro de los déficits sociales y el incremento de la pobreza, todo agravado con problemas de coherencia interna del diseño de estos programas.

El sesgo gerencial se evidencia en que , pese a los vacíos e inconsistencias de formulación, el PAMI no es un programa totalmente desatinado en su diseño, donde se le presenta como un programa básicamente de corte estructural-promocional. No obstante, los problemas de articulación, desfase intercomponentes (PAS, PEC, PAMI) y, en general de coordinación, lo desvian en la práctica de sus objetivos preventivos y educativos y lo convierten en un programa meramente asistencialista, como el resto.

Esto permite pensar que aún con la orientación compensatoria de las estrategias sociales, cónsona con el modelo neoliberal de desarrollo, el PAMI y otros programas similares podrian tener mejores resultados y ser de mediano impacto social, si se cuidaran y atacaran oportunamente los problemas que presentan en la práctica, especiaimente si se potenciara su componente educativo-formativo.
El problema de ignorancia está presente en dos planos: 1) en quienes toman las decisiones desde el más alto nivel gerencial, en tanto que: a. - demuestran un marcado desconocimiento de la realidad social venezolana y de la especificidad y complejidad del sector público social; b.* no están actualizados en el avance experimentado por conceptos como pobreza, desarrollo social, desarrollo humano, entre otros; c) desconocen metodologias de medición de impacto, de seguimiento, evaluacion, asi como estrategias gerenciales innovadoras. 2) Tambièn desde abajo: entre los be. neficiarios, quienes desconocen los programas, sus objetivos y ventajas, y actúan conformistamente, guiados por la cultura clientelar en ellos internalizada.

Todo esto indica que se precisa un proceso de capacitación, formación, educación y promoción, dirigido no sólo a los beneficiarios o usuarios de los programas y servicios sociales sino también hacia los gobernantes y hacia la alta y media gerencia social.

\section{Bibliografía citada}

Ahumads. Jorge $\$$ (!373). "Oterta y Demanda de Políticas Sociales: la percepción de los Beneficiarios". Ponencia presentada en el I Congreso Interamericano del CLAD sobre la Reforma del Estado y de la Administración Pública. Río de Janeiro, Brasil.

Alvarado, Neritza; Baralt, Leovic; Briceño, Alex; Diaz Irene y Ochoa, Elisabeth (1996). El Impacto de los Programas Sociales Compensatorios en el Zulia y Maracaibo, 1990-1995. Universidad del Zulia, Facultad de Ciencias Económicas y Sociales, Instituto de Investigaciones. Maracaibo.

Alvarado, Neritza (1997a). Factores Condicionantes del Impacto de la Política Social Venezolana: El Caso de la Estrategia PAMI en el Estado Zulia. Trabajo Libre de Investigación en el Doctorado en Estudios del Desarrollo 
del CENDES-UCV, presentado también como trabajo de Ascenso para optar a la Categoria de Profesor Asociado, Universidad del Zulia. Maracaibo. Venezuela.

Alvarado, Neritza (1997b). Evaluación del Impacto del Gasto Social sobre la Pobreza en Venezuela, 1979-1992. EDILUZ. Maracaibo. Venezuela.

BILBAO, Arantza y PACHANO, Susana (1998). "Programa Vaso de Vida". En: PNUD-BM. Alianzas para la Reducción de la Pobreza: Experiencias Exitosas en Venezuela. Henry Gómez Samper, Editor. Caracas.

Bustos, María I. (1998). Programas Sociales y Autoestima. Trabajo Final de Becaría de Investigación. Centro de Inves* tigaciones en Trabajo Social. Facultad de Clencias Jurídicas y Políticas. Universidad del Zulia. Maracaibo. Venezuela.

CONASSEPS (1994). Programa Ampliado Materno Infantil. Evaluación del Proceso. Informe Final. Caracas. Venezuela.

Franco, Rolando (1992). Las Nuevas Orientaciones de la Política Social en América Latina. Cuadernos Cátedra
Abierta. $N^{\circ} 3$. Fundación Escuela de Gerencia Social. Caracas.

Fonseca, Lady (1997). "Gerencia Social y Desarrollo". Ponencia presentada en el III Congreso Nacional de Trabajadores Sociales. Maracay, Venezuela.

Grindle, Marilú (1993). "Restricciones Políticas en la Implementación de Programas Sociales", En: Kliksberg, Bernardo (Compilador). Pobreza, un tema impostergable. FCE-CLAD-PNUD. Caracas. Venezuela.

Ministerio de Sanidad y Asistencia Social (MSAS), Fundación PAMI. Programa Ampliado Materno Infantil: Caracterización general de la estrategia (mimeo). Caracas. Venezuela.

Rodriguez, Aroldo (1983). Psicología Social. Editorial Trillas. 7 reimpresión. México.

Sulbrandt (1993)."La Evaluación de los Programas Sociales. Una Perspectiva Critica de los modelos Usuales", En: Kliksberg, B., compilador. Pobreza, un Tema impostergable. FCE. CLAD.PNUD. Caracas.

UCAB-MIN.FLIA. (1996). Encuesta de Opinión sobre los programas de la Agenda Venezuela. Intorme Ejecutivo. Caracas.

El Universal, 11-4 -98, p. D-3. Caracas. 